\title{
INJECTIVITY OF THE SYMMETRIC MAP FOR LINE BUNDLES
}

\author{
MONTSERRAT TEIXIDOR I BIGAS
}

\begin{abstract}
Let $C$ be a non-singular curve of genus $g$ defined over a field of characteristic different from two. We show that for every line bundle on $C$ of degree at most $g+1$, the natural product map $S^{2}\left(H^{0}(C, L)\right) \rightarrow H^{0}\left(C, L^{2}\right)$
\end{abstract}

is injective. We also show that the bound on the degree of $L$ is sharp

\section{INTRODUCTION}

Linear series have played a key role in the study of curves (and higher dimensional varieties). It is usually important to know how the spaces of sections of line bundles relate to each other. For example for a generic curve of genus $g$, the Petri map

$$
H^{0}(C, L) \otimes H^{0}\left(C, K \otimes L^{-1}\right) \rightarrow H^{0}(C, K)
$$

is injective. This property gives a lot of information on the structure of the set of linear series of a given dimension. On the other hand, the surjectivity of the maps

$$
S^{n}\left(H^{0}(C, L)\right) \rightarrow H^{0}\left(C, L^{n}\right)
$$

ensure the projective normality of a curve.

Despite its usefulness, there are few criteria that help in computing the rank of maps among various spaces of sections of vector bundles (see $[\mathrm{B}],[\mathrm{T} 2])$. The purpose of the following note is to help fill in this gap. We want to show that for a generic curve and any line bundle of degree at most $g+1$ with a given number of sections, the symmetric product map

$$
S^{2}\left(H^{0}(C, L)\right) \rightarrow H^{0}\left(C, L^{2}\right)
$$

is injective. The result is obviously false for line bundles of high degree, as even the dimension of the left hand side space becomes bigger than the dimension of the right hand side. We prove in fact that our bound is sharp: we construct an example of a line bundle of degree $g+2$ on a 
generic curve of genus $g$ (for every even $g \geq 4$ ) for which the symmetric product map is not injective (see 3.1).

Note that the natural product map

$$
m: H^{0}(C, L) \otimes H^{0}(C, L) \rightarrow H^{0}\left(C, L^{2}\right)
$$

is never injective for $k \geq 2$. Its kernel contains the subspace $\wedge^{2}\left(H^{0}(C, L)\right)$. Therefore, proving the injectivity of the map with domain in the symmetric power is equivalent to showing that the kernel of $m$ is precisely the wedge power. This kernel has proved to have important deformation theoretical meaning in particular cases (see for instance [T1]).

The main result of this paper is the following:

1.1. Theorem Let $C$ be a generic curve defined over an algebraically closed field of characteristic different from two. Let $L$ be a line bundle on $C$ such that $\operatorname{deg}(L)=d \leq g+1$. Then the map

$$
S^{2}\left(H^{0}(C, L)\right) \rightarrow H^{0}\left(C, L^{2}\right)
$$

is injective.

The proof of this result is inspired in the proof of the injectivity of the Petri map given by Eisenbud and Harris in [E,H2] (see also [W]).

\section{The CASE of Degree AT Most $g-1$}

We shall fix a genus $g$ and the degree $d$ for line bundles $L$ on $C$. We shall denote by $k$ (rather than the classical $r+1$ ) the dimension of the space of sections of these line bundles. We shall assume that $k, g, d$ have been fixed so that the generic curve of genus $g$ has line bundles of degree $d$ with $k$ sections (equivalently, the Brill- Noether number $g-k(g-1-d+k)$ is non-negative).

In order to prove 1.1 for a generic curve, it suffices to prove it for a special curve. Consider a family of curves $\pi: \mathcal{C} \rightarrow T$. Let $T$ be the spectrum of a discrete valuation ring $\mathcal{O}$ with maximal ideal generated by $t$. Assume that the generic fiber of $\pi$ is a non-singular curve and the special fiber $C$ looks as follows:

Take $g$ elliptic curves $E^{i}$ and let $P^{i}, Q^{i}$ be generic points on $E^{i}$. Take any number of rational curves $C_{1}^{0}, \ldots C_{k_{0}}^{0}, \ldots C_{0}^{g} \ldots C_{k_{q}}^{g}$ again with points $P_{j}^{i}, Q_{j}^{i}$ on them. Glue $C_{j}^{i}$ to $C_{j+1}^{i}$ by identifying $Q_{j}^{i}$ to $P_{j+1}^{i}$. Glue $C_{k_{i-1}}^{i-1}$ to $E^{i}$ by identifying $Q_{k_{i-1}}^{i-1}$ to $P^{i}$. Glue $E^{i}$ to $C_{1}^{i}$ by identifying $Q^{i}$ to $P_{1}^{i}$.

For convenience of notation, we shall denote by

$$
Y_{1}, \ldots Y_{M}, M=k_{0}+\ldots+k_{g}+g
$$


the components of $C$ starting with $C_{1}^{0}$ and ending with $C_{k_{g}}^{g}$. We shall denote by $P_{i}, Q_{i}$ the two points in $Y_{i}$ that get identified to $Q_{i-1} \in Y_{i-1}$ and $P_{i+1} \in Y_{i+1}$ respectively. We warn the reader that we shall keep the superindices $i$ when we need to refer to the $i^{\text {th }}$ elliptic curve. We hope this will not produce too much confusion.

Note that the form of the central fiber does not change if we make base changes and normalisations.

Consider now the set up of limit of a linear series in the sense of Eisenbud and Harris ([E,H2] p.273 or [E,H1], section 2). Let $\mathcal{L}$ be a line bundle on $\mathcal{C}$. One can modify $\mathcal{L}$ by tensoring with divisor with support on the central fiber. This leaves invariant the line bundle on the generic fiber but modifies it in the central fiber. For every component $Y_{i}$ of $C$, there is a line bundle $\mathcal{L}_{i}$ on $\mathcal{C}$ such that it has degree zero on every component of the central fiber except for the component $Y_{i}$. As $\mathcal{L}_{i}=\mathcal{L}_{i+1}\left(-d \sum_{j \leq i} Y_{j}\right)$, one can identify $\mathcal{L}_{i}$ with a subsheaf of $\mathcal{L}_{i+1}$.

Consider $\pi_{*}\left(\mathcal{L}_{i}\right)$ This is a free $\mathcal{O}$ module of rank k. Moreover, $\mathcal{L}_{i} \subset$ $\mathcal{L}_{i+1}$ is a lattice. Denote by $V_{i}$ the image of the restriction map

$$
\pi_{*} \mathcal{L}_{i} \rightarrow \pi_{*} \mathcal{L}_{i \mid Y_{i}}=H^{0}\left(Y_{i}, \mathcal{L}_{i \mid Y_{i}}\right)
$$

As $\operatorname{deg} \mathcal{L}_{i \mid Y_{j}}=0, j \neq i$, this map is injective and we shall sometimes identify $\pi_{*}\left(\mathcal{L}_{i}\right)$ with $V_{i}$.

From [E,H2] Lemma 1.2, one can find a basis of sections $\sigma_{m}^{i}, m=$ $1 . . k$ of the free module $\pi_{*}\left(\mathcal{L}_{i}\right)$ such that the orders of vanishing of the sections $\sigma_{m}^{i}$ at $P_{i}$ are the different orders of vanishing of the sections of $V_{i}$ and $t^{\alpha_{m}^{i}} \sigma_{m}^{i}, m=1 \ldots k$ form a basis for $\pi_{*} \mathcal{L}_{i+1}$.

We shall now relate the vanishing of sections of line bundles at the various nodes.

2.1. Lemma 1) Let $Y$ be any irreducible non-singular curve $L$ a line bundle of degree $d$ on $Y$ and $P, Q$ two points on $Y$. The sum of the orders of vanishing at $P$ and $Q$ of any section of $L$ is at most $d$.

2) Let $Y$ be an elliptic curve and $P, Q$ generic points of $Y$. Let $L$ be a line bundle of degree $d$ on $Y$.The sum of the orders of vanishing at $P$ and $Q$ of any section of $L$ is at most $d-1$ except in the case where $L=\mathcal{O}(a P+(d-a) Q)$ for some a. In this case, there is only one section of $L$ vanishing with multiplicities adding up to $d$ at the two points.

Proof. If a section of a line bundle $L$ vanishes with order $a$ at $P$ and $b$ at $Q$, then $\mathcal{O}(a P+b Q)$ is a subsheaf of $L$. Hence, $d \geq a+b$. This proves the first statement.

If $a+b=d$, then $L=\mathcal{O}(a P+b Q)$. If there is another section vanishing to orders $a^{\prime}, b^{\prime}$ at $P, Q$ with $a^{\prime}+b^{\prime}=d$ and say $a^{\prime}>a$ then 
$a P+b Q \equiv a^{\prime} P+b^{\prime} Q$. Hence, $c P \equiv c Q$ with $c=a^{\prime}-a$. This contradicts the genericity of the pair $P, Q$ if $Y$ is not rational.

Remark The genericity of $P, Q$ is essential here. If $c P$ is linearly equivalent to $c Q$ for some $c \leq d$, then the line bundle $\mathcal{O}(a P+(d-$ a) $Q$ ), $a \geq c$ has (at least) two sections with orders of vanishing adding up to $d$ at $P, Q$ namely $a P+(d-a) Q$ and $(a-c) P+(d-a+c) Q$.

The following result of Eisenbud and Harris (cf. Prop 1.1 in $[\mathrm{E}, \mathrm{H}]$ ), will be used in the sequel.

2.2. Lemma Let $\sigma$ be a section in $\pi_{*} \mathcal{L}_{i}$. Let $\alpha$ be the unique integer such that $t^{\alpha} \sigma \in \pi_{*}\left(\mathcal{L}_{i+1}\right)-t \pi_{*}\left(\mathcal{L}_{i+1}\right)$, then

$$
\operatorname{ord}_{P_{i}}\left(\sigma_{\mid Y_{i}}\right) \leq d-\operatorname{ord}_{Q_{i}}\left(\sigma_{\mid Y_{i}}\right) \leq \alpha \leq \operatorname{ord}_{P_{i+1}}\left(t^{\alpha} \sigma_{\mid Y_{i+1}}\right)
$$

As in [E,H], p.277, one can define the order of a section $\rho \in S^{2}\left(\pi_{*} \mathcal{L}_{Y}\right)$ at a point $P$ in a component $Y$ as follows:

2.3. Definition $W e$ say $\operatorname{ord}_{P}\left(\rho_{\mid Y}\right) \geq l$ if and only if $\rho$ is in the linear span of $t\left(S^{2}\left(\pi_{*}\left(\mathcal{L}_{Y}\right)\right)\right)$ and elements of the form $\sigma_{m} \otimes \sigma_{n}+\sigma_{n} \otimes \sigma_{m}$ where $\operatorname{ord}_{P}\left(\sigma_{n}\right)+\operatorname{ord}_{P}\left(\sigma_{m}\right) \geq l, \sigma_{n}, \sigma_{m} \in \pi_{*}\left(\mathcal{L}_{Y}\right)$.

One then has the following result (cf. [E,H2], Lemma 3.2)

2.4. Lemma Let $\sigma_{m}$ be a basis of the free $\mathcal{O}$ module $\pi_{*}\left(\mathcal{L}_{i}\right)$ such that the orders of vanishing of the $\sigma_{m}$ at $P_{i}$ are the distinct orders of vanishing of the linear series at this point and $t^{\alpha_{m}} \sigma_{m}$ is a basis of $\pi_{*}\left(\mathcal{L}_{i+1}\right)$. If

$$
\rho=\sum f_{n, m}\left(\sigma_{n} \otimes \sigma_{m}+\sigma_{m} \otimes \sigma_{n}\right) \in S^{2}\left(\pi_{*} \mathcal{L}_{i}\right)
$$

where the $f_{n, m}$ are functions on the discrete valuation ring $\mathcal{O}$ and the associated discrete valuation is $\nu$, then

$$
\operatorname{ord}_{P_{i}}\left(\rho_{\mid Y_{i}}\right)=\min _{\left\{\nu\left(f_{n, m}\right)=0\right\}}\left(\operatorname{ord}_{P_{i}}\left(\sigma_{n}\right)+\operatorname{ord}_{P_{i}}\left(\sigma_{m}\right)\right)
$$

If $\beta$ is the unique integer such that

$$
t^{\beta} \rho \in S^{2} \pi_{*} \mathcal{L}_{i+1}-t\left(S^{2} \pi_{*} \mathcal{L}_{i+1}\right)
$$

then

$$
\beta=\max \left\{\alpha_{n}+\alpha_{m}-\nu\left(f_{n m}\right)\right\}
$$

Let us assume now that the kernel of the symmetric product map is non-zero on the generic curve. We can then find an element $\rho$ such that say $\rho \in S^{2}\left(\pi_{*} \mathcal{L}_{1}\right)-t S^{2}\left(\pi_{*} \mathcal{L}_{1}\right)$ and integers $\beta_{i}, i=2 \ldots M$ such that $t^{\beta_{i}} \rho \in S^{2}\left(\pi_{*} \mathcal{L}_{i}\right)-t S^{2}\left(\pi_{*} \mathcal{L}_{i}\right)$ and $t^{\beta_{i}} \rho \in \operatorname{Ker}\left(S^{2}\left(\pi_{*} \mathcal{L}_{i}\right) \rightarrow \pi_{*}\left(\mathcal{L}_{i}\right)^{2}\right)$.

As a section of a line bundle cannot vanish to order higher than the degree, the following claim will conclude the proof in the case $d \leq g-1$. 
2.5. Claim If $l>k_{0}+\ldots+k_{m-1}+m$, then ord $_{P_{l}}\left(t^{\beta_{l}} \rho\right) \geq 2 m$. In particular, for $l \geq k_{0}+\ldots+k_{g-1}+g$, $\operatorname{ord}_{P_{l}}\left(t^{\beta_{l}} \rho\right) \geq 2 g$.

Proof. We prove the following two statements:

1) If $C_{i}$ is a rational curve,

$$
\operatorname{ord}_{P_{i+1}}\left(t^{\beta_{i+1}} \rho_{\mid Y_{i+1}}\right) \geq \operatorname{ord}_{P_{i}}\left(t^{\beta_{i}} \rho_{\mid Y_{i}}\right)
$$

2) If $C_{i}$ is an elliptic component,

$$
\operatorname{ord}_{P_{i+1}}\left(t^{\beta_{i+1}} \rho_{\mid Y_{i+1}}\right) \geq \operatorname{ord}_{P_{i}}\left(t^{\beta_{i}} \rho_{\mid Y_{i}}\right)+2
$$

In other words, the order of vanishing of a section $t^{\beta_{i+1}} \rho$ at $P_{i+1}$ is at least as large as that of $t^{\beta_{i}} \rho$ at $P_{i}$ if $Y_{i}$ is rational and at least two units larger if it is elliptic. As the order of vanishing $t^{\beta_{0}} \rho$ at $P_{0}$ is nonnegative, this shows that the order of vanishing of $t^{\beta_{i}} \rho$ at $P_{i}$ is at least $2 m$ if $m$ elliptic curves precede $Y_{i}$. This is the first part of the claim. The second part follows from the first when $m=g$.

We now turn to the proof of 1) and 2). Choose a basis $\sigma_{m}, m=$ $1 \ldots k$ of $\pi_{*}\left(\mathcal{L}_{i}\right)$ such that $t^{\alpha_{m}} \sigma_{m}$ is a basis of $\pi_{*} \mathcal{L}_{i+1}$. For simplicity of notation, we shall assume that $\beta_{i}=0$ Write

$$
\rho=\sum_{n \leq m} f_{n m}\left(\sigma_{n} \otimes \sigma_{m}+\sigma_{m} \otimes \sigma_{n}\right) \in S^{2}\left(\pi_{*} \mathcal{L}_{i}\right)
$$

Then, from 2.4,

$$
\operatorname{ord}_{P_{i}}\left(\rho_{\mid Y_{i}}\right)=\min _{\left\{\nu\left(f_{n m}\right)=0\right\}}\left(\operatorname{ord}_{P_{i}}\left(\sigma_{n}\right)+\operatorname{ord}_{P_{i}}\left(\sigma_{m}\right)\right)
$$

Assume that this minimum is attained by a pair corresponding to the indices $n_{0}, m_{0}$ with $\nu\left(f_{n_{0}, m_{0}}\right)=0$. Then from 2.2 ,

$\left.\operatorname{cod}_{P_{i}}\left(\sigma_{n_{0}}\right)+\operatorname{ord}_{P_{i}}\left(\sigma_{m_{0}}\right)\right) \leq 2 d-\operatorname{ord}_{Q_{i}}\left(\sigma_{n_{0}}\right)-\operatorname{ord}_{Q_{i}}\left(\sigma_{m_{0}}\right) \leq \alpha_{n_{0}}+\alpha_{m_{0}}$

From 2.4 and the fact that $\nu\left(f_{n_{0}, m_{0}}\right)=0$, the latter is at most $\beta_{i+1}$.

Write

$$
t^{\beta_{i+1}} \rho=\sum_{n \leq m}\left(t^{\beta_{i+1}-\alpha_{n}-\alpha_{m}} f_{n m}\right)\left(t^{\alpha_{n}} \sigma_{n} \otimes t^{\alpha_{m}} \sigma_{m}+t^{\alpha_{m}} \sigma_{m} \otimes t^{\alpha_{n}} \sigma_{n}\right)
$$

Hence, from 2.4

$\operatorname{ord}_{P_{i+1}}\left(t^{\beta_{i+1}} \rho_{\mid Y_{i+1}}\right)=\min _{\left\{\beta_{i+1}-\alpha_{n}-\alpha_{m}+\nu\left(f_{n m}\right)=0\right\}}\left(\operatorname{ord}_{P_{i+1}}\left(t^{\alpha_{n}} \sigma_{n}\right)+\operatorname{ord}_{P_{i+1}}\left(t^{\alpha_{m}} \sigma_{m}\right)\right)$

Assume that this minimum is attained at a pair $n_{1}, m_{1}$ with

$$
\beta_{i+1}-\alpha_{n_{1}}-\alpha_{m_{1}}+\nu\left(f_{n_{1}, m_{1}}\right)=0
$$

Then,

$\left.\beta_{i+1} \leq \beta_{i+1}+\nu\left(f_{n_{1}, m_{1}}\right)=\alpha_{n_{1}}+\alpha_{m_{1}} \leq \operatorname{ord}_{P_{i+1}}\left(t^{\alpha_{n_{1}}} \sigma_{n_{1}}\right)+\operatorname{ord}_{P_{i+1}}\left(t^{\alpha_{m_{1}}} \sigma_{m_{1}}\right)\right)$

where the last inequality comes from 2.2 
Stringing together the above inequalities, we obtain $\operatorname{ord}_{P_{i}}\left(\rho_{\mid Y_{i}}\right) \leq$ $\operatorname{ord}_{P_{i+1}}\left(\rho_{\mid Y_{i+1}}\right)$. Hence part 1) is proved.

Assume now that there is equality in the inequality above. Then all the previous inequalities must be equalities. In particular, the terms $\sigma_{n_{0}} \otimes \sigma_{m_{0}}+\sigma_{m_{0}} \otimes \sigma_{n_{0}}$ that give the vanishing of $\rho$ at $P_{i}$ satisfy

$$
\operatorname{ord}_{P_{i}}\left(\sigma_{k}\right)+\operatorname{ord}_{Q_{i}}\left(\sigma_{k}\right)=d, k=n_{0}, m_{0}
$$

If we have $\operatorname{ord}_{P_{i+1}}\left(\rho_{\mid Y_{i+1}}\right)=\operatorname{ord}_{P_{i}}\left(\rho_{\mid Y_{i}}\right)+1$, then in each pair, one of $\sigma_{k}, k=n_{0}, m_{0}$ would vanish to order $d$ between the two nodes. From 2.1 , there is at most one such section $\sigma_{i_{0}}$ on our elliptic curves.

Hence, if $\operatorname{ord}_{P_{i+1}}\left(\rho_{\mid Y_{i+1}}\right) \leq \operatorname{ord}_{P_{i}}\left(\rho_{\mid Y_{i}}\right)+1$, the terms in $\rho$ giving the vanishing at $P_{i}$ could be written as

$$
\sigma_{i_{0}} \otimes \sigma+\sigma \otimes \sigma_{i_{0}}
$$

for some section $\sigma$. As a section like this cannot be in the kernel of the symmetric product map, this concludes the proof of the claim.

\section{The CASES of Degree $g, g+1$ And COUnterexamples in DEGREE $g+2$}

Assume now $d=g$. Consider the last elliptic component $E^{g}$ with nodes $P^{g}, Q^{g}$. Write the restriction to the central fiber of an element in the kernel of the symmetric evaluation map as

$$
\rho=\sum f_{n m}\left(\sigma_{n} \otimes \sigma_{m}+\sigma_{m} \otimes \sigma_{n}\right), \sigma_{n}, \sigma_{m} \in \pi_{*}\left(\mathcal{L}_{E^{g}}\right)
$$

Consider a pair $\sigma_{n}, \sigma_{m}$ that gives the vanishing of $\rho$ at $P^{g}$. From 2.5, $\operatorname{ord}_{P^{g}}\left(\sigma_{n}\right)+\operatorname{ord}_{P^{g}}\left(\sigma_{m}\right) \geq 2(g-1)$. As $\operatorname{deg}\left(\mathcal{L}_{E^{g} \mid E^{g}}\right)=g, \operatorname{ord}_{P^{g}}\left(\sigma_{k}\right) \leq$ $g, k=n, m$. It follows that the possible orders of such sections are $g, g-1, g-2$. If there is a section vanishing to order $g$ at $P^{g}$, then $\mathcal{L}_{E^{g} E^{g}}=\mathcal{O}\left(g P^{g}\right)$ and this line bundle does not have any section vanishing to order $g-1$ at $P^{g}$. Hence there are at most two independent sections among the $\sigma_{k}$ and for every pair that appears, the sum of the orders of vanishing at $P^{g}$ is the same. But then $\rho$ cannot be in the kernel of the symmetric product map. This concludes the proof for $d=g$

Assume now that $d=g+1$. If $\operatorname{ord}_{P^{1}}(\rho)=0,1$, then the pairs of sections giving the vanishing at $P^{1}$ will vanish to orders 0,1 . From our choice of basis, there are at most two sections satisfying these conditions. Hence, again $\rho$ could not be in the kernel of the symmetric product map. Therefore the vanishing of $\rho$ at $P^{1}$ is at least two. From 
statements 1) and 2) in 2.5, the vanishing at $P^{g}$ is at least $2 g$. Now, we conclude as in the case $d=g$.

3.1. Example If $g$ is even, $g \geq 4$ a generic curve of genus $g$ has a line bundle of degree $g+2$ such that the kernel of the symmetric product map is not injective.

Proof. A generic curve of even genus $g$ has line bundles of degree $g / 2+1$ with two sections (because the corresponding Brill-Noether number is zero). Take two different ones $L_{1}, L_{2}$ and choose independent sections $s_{1}, t_{1}$ and $s_{2}, t_{2}$ of them. Take $L=L_{1} \otimes L_{2}$. This is a line bundle of degree $g+2$ that contains the sections $s_{1} s_{2}, s_{1} t_{2}, t_{1} s_{2}, t_{1} t_{2}$. These are four independent sections, as can be checked using the base point free pencil trick.

Consider now the following section

$$
\left(s_{1} s_{2} \otimes t_{1} t_{2}+t_{1} t_{2} \otimes s_{1} s_{2}\right)-\left(s_{1} t_{2} \otimes s_{2} t_{1}+s_{2} t_{1} \otimes s_{1} t_{2}\right)
$$

This section is non-zero and in the kernel of the symmetric product map.

\section{REFERENCES}

[B] D.Butler normal generation of vector bundles over a curve J.Diff.Geom 39 (1994), 1-34.

[E,H1] D.Eisenbud, J.Harris Limit linear series, basic theory, Invent.Math. 85 (1986), 337-371.

[E,H2] D.Eisenbud, J.Harris A simpler proof of the Gieseker-Petri Theorem for special divisors, Invent.Math. 74 (1983), 269-280.

[T1] M.Teixidor Half-canonical series on algebraic curves Trans.AMS 302 (1987), 99-115.

[T2] M.Teixidor Curves in Grassmannians Proc.AMS 126 (1998), 1597-1603.

[W] G.Welters A Theorem of Gieseker-Petri type for Prym varieties Ann.Sci. Ec. Norm. 4s., t 18, 1985, 671-683

Mathematics Department, Tufts University, Medford, MA02155

E-mail address: montserrat.teixidoribigas@tufts.edu 